\title{
Seroprevalencia de Toxoplasma gondii en un hospital universitario en Chile
}

\author{
Toxoplasma gondii seroprevalence in a university hospital in Chile
}

\author{
Natalia Covarrubias', Daniela B. Vera' y Carmen Hurtado
}

`Sección de Gastroenterología, Departamento de Medicina Interna, Hospital Clínico Universidad de Chile, Santiago, Chile.

Conflictos de interés: las autoras declaran que no existen conflictos de interés.

Fuente de Financiamiento: no existen fuentes de financiamiento.

Recibido: 7 de agosto de 2020 / Aceptado: 20 de noviembre de 2020

\section{Resumen}

La toxoplasmosis es una enfermedad zoonótica causada por Toxoplasma gondii. Se estima que afecta a un tercio de la población mundial. En Chile, en 1996, se reportó que la seroprevalencia de anticuerpos del tipo IgG contra $T$. gondii fue $36,9 \%$, la cual aumentaba progresivamente de norte a sur del país. Hasta el momento, no existe información actualizada sobre la seroprevalencia de $T$. gondii en la Región Metropolitana. En el presente estudio, se determinó la seroprevalencia de IgG anti T. gondii en el periodo 2013-2018 en un centro universitario de Santiago. De un total de 1.666 resultados, 386 $(23,2 \%)$ fueron positivos. No se encontraron diferencias según sexo y hubo un incremento significativo según el rango etario. No se observó una disminución de la seroprevalencia en los últimos seis años; sin embargo, los resultados señalan una reducción significativa con respecto a investigaciones previas realizadas en la Región Metropolitana.

Palabras clave: Toxoplasmosis; seroprevalencia; anticuerpos IgG.

\section{Introducción}

L a toxoplasmosis es una enfermedad zoonótica causada por Toxoplasma gondii, un parásito protozoo intracelular ampliamente hospedero definitivo y una amplia variedad de potenciales hospederos intermediarios, tales como: humanos, mamíferos no felinos, aves, animales domésticos o de granja, entre otros ${ }^{1}$.

Los modos de transmisión más relevantes en el ser humano son: vía congénita (transmisión vertical), consumo de carnes infectadas con bradizoítos, deglución de ooquistes del ambiente por medio de alimentos o agua contaminada con materia fecal de gatos y por medio de trasplante

\begin{abstract}
Toxoplasmosis is a zoonotic disease caused by Toxoplasma gondii. It is estimated to affect a third of the world's population. In Chile, in 1996, a seroprevalence of IgG antibodies against T. gondii of $36.9 \%$ was reported, which progressively increased from north to south of the country. There are no updated reports of the seroprevalence of $T$. gondii in the Metropolitan Region. In the present study, we determined the seroprevalence of anti $T$. gondii $\operatorname{IgG}$ in the 2013-2018 period at the Clinical Hospital University of Chile. Of a total of 1,666 results, $386(23.2 \%)$ were positive, without gender differences, but with a significant increase with age. A decrease in seroprevalence was not observed in the last six years, however the results obtained show a significant reduction compared to previous research carried out in the Metropolitan Region.

Key words: Toxoplasmosis; seroprevalence; IgG antibody.
\end{abstract}

de órganos infectados y/o transfusiones con quistes o zoítos de T. gondii ${ }^{2}$.

Los factores que contribuyen a la adquisición de la infección son los hábitos higiénicos-dietéticos, tales como el consumo de carnes poco cocidas, verduras mal lavadas, falta de higiene en la manipulación de los alimentos, un clima cálido y húmedo, no tener acceso a agua potable y la presencia de gatos ${ }^{3}$.

En general, la toxoplasmosis es una infección que cursa de forma asintomática y autolimitada en individuos inmunocompetentes, mientras que puede llegar a causar complicaciones y daños serios al feto si la mujer se infecta durante el embarazo, así como la infección grave en pacientes inmunocomprometidos ${ }^{2,4}$.

Se estima que la toxoplasmosis afecta a un tercio de la población 
mundial. En los E. U. A. es considerada una de las principales causas de hospitalización y muerte atribuida a enfermedades transmitidas por los alimentos ${ }^{3,5}$. La distribución de la infección en todo el mundo es variable, incluso dentro de un país, probablemente debido a diferencias ambientales, tales como saneamiento, clima y humedad; socio-económicas y culturales de un lugar en particular y sus residentes, con tasas de prevalencia en los países en desarrollo que varían entre 30 y $60 \% \%^{2,6}$.

En Chile, la toxoplasmosis es endémica, presentando amplias diferencias regionales. Un estudio del Departamento de Microbiología y Parasitología de la Facultad de Medicina de la Universidad de Chile, publicado en 1996, realizado en 76.317 personas de diferentes edades, estableció una seroprevalencia de anticuerpos del tipo IgG contra $T$. gondii de $36,9 \%$ (n $=28.124$ ), la cual aumentaba progresivamente de norte a sur del país ${ }^{7}$. El estudio más reciente en nuestro país reportó una seroprevalencia de $55,9 \%$ en la Región de Los Ríos ${ }^{8}$. Hasta el momento, no existe información actualizada de la seroprevalencia de $T$. gondii en la Región Metropolitana.

La vigilancia diaria de la detección de anticuerpos anti-Toxoplasma en nuestro centro, nos ha permitido una recopilación continua de resultados de una amplia variedad de sujetos, utilizando el mismo kit comercial. El objetivo del estudio fue determinar la seroprevalencia de $T$. gondii en el periodo 2013-2018 en un centro de referencia ubicado en Santiago, Chile.

\section{Materiales y Métodos}

\section{Pacientes y métodos}

Se recolectaron 1.666 resultados con análisis de $\operatorname{IgG}$ anti- $T$. gondii desde enero de 2013 hasta diciembre de 2018, a partir de exámenes con solicitud de medición de anticuerpos IgG anti- $T$. gondii en el Laboratorio de Gastroenterología del Hospital Clínico de la Universidad de Chile. Si el paciente presentaba más de una prueba disponible, se consideró sólo el primer resultado.

La totalidad de las determinaciones fueron realizadas con técnica ELFA, mini VIDAS ${ }^{\circledR}$ TOXO IgG II (BioMérieux SA, Francia). Se consideraron positivas las muestras con títulos $\geq 8 \mathrm{UI} / \mathrm{ml}$, según instrucciones del fabricante. Los datos demográficos disponibles fueron edad y sexo. La asociación entre las variables de estudio y la seropositividad de IgG anti-T. gondii se estimó mediante el test de chi cuadrado. Un $\mathrm{p}<0,05$ fue considerado estadísticamente significativo. El estudio fue aprobado por el comité de ética local.

\section{Análisis estadísticos}

Las variables categóricas fueron expresadas como valor absoluto y porcentaje, mientras que las variables cuantitativas, como media \pm desviación estándar. Se utilizó el test de proporciones chi-cuadrado $(\mathrm{p}<0,05)$.

Los análisis fueron realizados con el software estadístico SPSS IBM Corp. Released 2016. IBM SPSS Statistics for Windows, Version 24.0. Armonk, NY: IBM Corp.

\section{Resultados}

Se analizaron un total de 1.666 resultados, correspondientes a 962 $(57,7 \%)$ hombres y $704(42,3 \%)$ mujeres (relación M/F: 1,4) y la edad promedio fue 37 años (rango, 0-88 años). En total, 1.240 (74,4\%) resultados fueron negativos, $386(23,2 \%)$ positivos y $40(2,4 \%)$ dudosos. $\mathrm{La}$

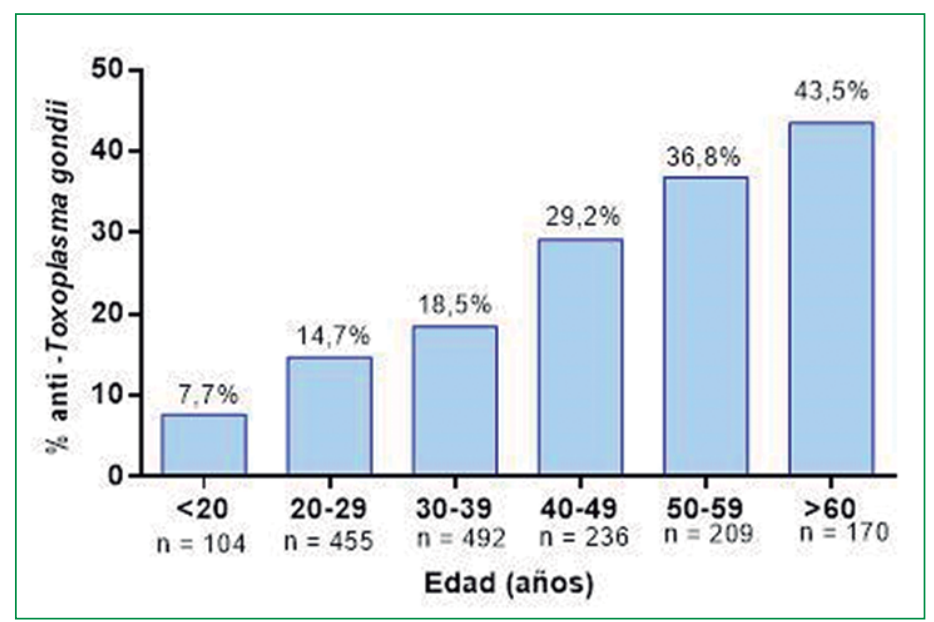

Figura 1. Seroprevalencia de anticuerpos $\lg G$ anti-Toxoplasma gondii por grupo de edad.

\begin{tabular}{lccccccc}
$\begin{array}{l}\text { Tabla 1. Seroprevalencia de anticuerpos } \\
\text { por año y sexo }\end{array}$ \\
\hline Año & $\mathbf{2 0 1 3}$ & $\mathbf{2 0 1 4}$ & $\mathbf{2 0 1 5}$ & $\mathbf{2 0 1 6}$ & $\mathbf{2 0 1 7}$ & $\mathbf{2 0 1 8}$ \\
\hline Masculino (n) & 144 & 141 & 145 & 139 & 171 & 222 \\
\hline Prevalencia (\%) & 27,1 & 25,5 & 24,8 & 27,3 & 21,6 & 17,6 \\
\hline Femenino (n) & 132 & 111 & 84 & 102 & 131 & 144 \\
\hline Prevalencia (\%) & 21,2 & 25,2 & 16,7 & 20,6 & 27,5 & 23,6 \\
\hline Total & 276 & 252 & 229 & 241 & 302 & 366 \\
\hline Prevalencia (\%) & 24,2 & 25,3 & 21,8 & 24,5 & 24,2 & 19,9 \\
\hline
\end{tabular}

seroprevalencia aumentó significativamente con la edad de los pacientes, de $7,7 \%$ en menores de 20 años a $43,5 \%$ en mayores de 59 años $(\mathrm{p}<0,0001)$ (Figura 1). Por otro lado, la seroprevalencia mostró una tendencia a disminuir progresivamente en el tiempo, entre 2013 y 2018, la cual no fue estadísticamente significativa: de 24,2\% en 2013 a 19,9\% en 2018 (Tabla 1). No hubo diferencias significativas en cuanto a la seropositividad entre hombres y mujeres $(23,4$ y $22,9 \%$; respectivamente).

\section{Discusión}

En el presente estudio se encontró una seroprevalencia de anticuerpos IgG anti $T$. gondii de 23,2\%, una de las más bajas reportadas en Latinoamérica $^{6}$. No se observó una disminución significativa de la seroprevalencia en los últimos seis años; sin embargo, los resultados obtenidos muestran una reducción con respecto a investigaciones previas realizadas en la Región Metropolitana. Es así como un estudio realizado entre el año 1982 y 1994 , reportó 33,3\% de seroprevalencia en 13.637 sujetos, cifra significativamente superior a lo encontrado en el presente estudio ${ }^{7}$. Asimismo, si se compara el intervalo de edad de 0-30 años estudiado en 1996, en 53.638 individuos de todo Chile y en 1997, en 560 sujetos de la Región Metropolitana, con el mismo rango etario de la presente investigación $(n=559)$, nuestros 
resultados de seropositividad $(13,4 \%)$ son significativamente menores a los obtenidos por estos autores (34,3 y $24,6 \%$; respectivamente) $)^{7,9}$.

La infección por $T$. gondii está sujeta a múltiples factores (ambientales, socioeconómicos, higiene, alimentarios, entre otros), los cuales pueden tener variaciones en el tiempo. En concordancia con nuestros datos, una disminución en la seroprevalencia se ha reportado en varios países, independientemente de la cifra inicial ${ }^{10-13}$. Jones y cols., compararon la seroprevalencia en la población de E.U.A. en los períodos comprendidos entre 1988-1994 y 2009-2010 y observaron una disminución de 14,1 a $6,7 \%{ }^{10}$. Un estudio realizado en el norte de Grecia informó una baja continua, de 37 a 29,9 y 24,1\% en 1984, 1994 y 2004; respectivamente ${ }^{11}$. En Francia, un estudio realizado en mujeres embarazadas observó entre 1995 y 2006, un descenso de 35,2 a 18,5\% ${ }^{12}$. En Latinoamérica, específicamente en Argentina, la seroprevalencia en los donantes de sangre disminuyó del $67 \%$ en 1967 al $21,2 \%$ en $2017^{3}$.

Actualmente, no existe una explicación concluyente para esta disminución, cada país lo atribuye a distintos factores según su realidad local. En general, los argumentos podrían asociarse a mejores condiciones sanitarias, el acceso a agua potable, mejores procesos de calidad en la industria alimentaria y el consumo de alimentos congelados o cocidos que reducen la posibilidad de infección. Es decir, múltiples variables que influyen en la disminución de la tasa de exposición de individuos susceptibles a quistes tisulares o reservorios ambientales contaminados.

Es importante mencionar, que una situación distinta es la descrita en el sur de Chile, específicamente en la Región de los Ríos, en donde en el año 2016 se reportó un aumento significativo con respecto a estudios previos $^{8}$, encontrándose una seroprevalencia de toxoplasmosis de $55 \%$, la cual fue superior a la estimación de $47 \%$ obtenida de 6.438 personas realizada en $1990^{14}$, ambas cifras muy superiores a lo encontrado en la Región Metropolitana. La mayor prevalencia en el sur, ha sido relacionada con las condiciones ambientales y una mayor producción y consumo de diferentes tipos de carne. El aumento indica que no se han logrado mejoras en términos de programas de prevención y reducción de infecciones ${ }^{8,14}$.

El gato, como hospedero definitivo, cumple un rol importante en la difusión del parásito en la naturaleza. Un estudio realizado en la Región Metropolitana en 230 gatos, encontró una prevalencia de $4,3 \%{ }^{15}$, mientras que en la Región de los Ríos se encontró una prevalencia de $33 \%$ en 97 gatos $^{16}$. Lo anterior podría explicar el mayor reservorio que existe en esa región, lo cual se correlaciona con las distintas seroprevalencias encontradas en nuestro país. Una de las explicaciones de esta diferencia, está dada porque la mayoría de los gatos caseros de grandes ciudades son alimentados con productos concentrados comerciales. Esto podría estar disminuyendo la seropositividad de los mismos contra $T$. gondii, al no verse obligados a cazar (evitando así su infección por medio de quistes tisulares) y disminuyendo la liberación de ooquistes al medio externo a través de las heces.

El aumento significativo de la seroprevalencia de la toxoplasmosis con la edad se explica porque con el tiempo aumentan las posibilidades de contacto directo o alimentario con las formas infectantes (ooquistes y bradizoitos) $)^{7,8,13}$. Este hecho fue corroborado en el presente estudio donde la mayor proporción de seropositividad se encontró en el grupo de sujetos mayores de 59 años $(43,5 \%)$.

Actualmente, en nuestro país el control prenatal en mujeres embarazadas, realizado en los centros de salud, está dirigido a descartar la infección por VIH, sífilis en todo el país y Chagas en regiones de alta endemia, no considerando a la toxoplasmosis como una enfermedad de importancia.
No existe una mayor sensibilización acerca de esta enfermedad en el país, a pesar de la experiencia internacional que da cuenta de la modificación de la presentación clínica y su potencial asociación con otras patologías 2 .

En consecuencia, la presente investigación muestra que los valores de seropositividad para $T$. gondii han disminuido de manera importante en los últimos 30 años en la Región Metropolitana, lo cual incrementa el número de personas susceptibles a la infección. Esto es de particular importancia en individuos con algún tipo de inmunosupresión y en aquellas mujeres seronegativas en edad fértil que corren el riesgo de adquirir la primoinfección por $T$. gondii durante el primer trimestre del embarazo. Nuestros resultados no son representativos de todo el país, por lo que se requieren más estudios para establecer la situación del país en relación a esta zoonosis y profundizar su posible efecto en la salud pública. En paralelo, también es importante informar y educar a la población, especialmente la de riesgo, sobre cómo prevenir la infección.

\section{Referencias bibliográficas}

1.- Montoya JG, Liesenfeld O. Toxoplasmosis. Lancet 2004; 363: 1965-76. doi: 10.1016/S0140-6736(04)16412-X.

2.- Mimica F, Muñoz-Zanzi C, Torres M, Padilla O. Toxoplasmosis, zoonosis parasitaria prevalente en Chile: recuento y desafíos. Rev Chilena Infectol 2015; 32: 541-9. http://dx.doi.org/10.4067/S0716-10182015000600008.

3.- Kaufer FJ, Carral LA, Messina MT, Schneider MV, Mendez M, Herrera L, et al. Prevalencia de anticuerpos anti-Toxoplasma gondii en hemodonantes en la ciudad de Buenos Aires desde 1967 a 2017. Medicina (Buenos Aires) 2017; 77: 475-80.

4.- Robert-Gangneux F, Dardé ML. Epidemiology of and diagnostic strategies for toxoplasmosis. Clin Microbiol Rev 2012; 25: 264-96. doi: 10.1128/ CMR.05013-11.

5.- Jones JL, Dubey JP. Foodborne toxoplasmosis. Clin Infect Dis 2012; 55 : 845-51. doi: 10.1093/cid/cis508.

6.- Foroutan-Rad M, Majidiani H, Dalvand S, Daryani A, Kooti W, Saki J, et al. Toxoplasmosis in blood donors: a systematic review and meta-analysis. Transfus Med Rev 2016; 30: 116-22. doi: 10.1016/j. tmrv.2016.03.002.

7.- Contreras M C, Schenone H, Salinas P, Sandoval L, Rojas A, Villarroel F, et al. Seroepidemiology of human toxoplasmosis in Chile. Rev Inst Med Trop S. Paulo 1996; 38: 431-5. doi: 10.1590/s0036-46651996000600008.

8.- Munoz-Zanzi C, Campbell C, Berg S. Seroepidemiology of toxoplasmosis in rural and urban communities from Los Rios Region, Chile. Infect Ecol Epidemiol 2016; 6: 10.3402. doi: 10.3402/iee.v6.30597.

9.- Abarca K, Vial P, Zamorano J, Paris C, Ferres M, Villaroel L, et al. Seroprevalence of cytomegalovirus and Toxoplasma gondii in healthy subjects under 30 years old in Santiago, Chile. Rev Med Chile 1997; 125 : 531-8.

10.- Jones JL, Kruszon-Moran D, Rivera HN, Price C, Wilkins PP. Toxoplasma gondii seroprevalence in the United States 2009-2010 and comparison with the past two decades. Am J Trop Med Hyg 2014; 90: 1135-9. doi: 10.4269/ ajtmh.14-0013.

11.- Diza E, Frantzidou F, Souliou E, Arvanitidou M, Gioula G, Antoniadis A. Seroprevalence of Toxoplasma gondii in northern Greece during the last 20 years. Clin Microbiol Infect 2005; 11: 719-23. doi: 10.1111/j.14690691.2005.01193.x.

12.- Hofhuis A, van Pelt W, van Duynhoven YT, Nijhuis CD, Mollema L, van der Klis FR, et al. Decreased prevalence and age-specific risk factors for Toxoplasma gondii IgG antibodies in The Netherlands between 1995/1996 and 2006/2007. Epidemiol Infect 2011; 139: 530-8. doi: 10.1017/ S0950268810001044.

13.- Guigue N, Léon L, Hamane S, Gits-Muselli M, Le Strat Y, Alanio A. 


\section{Comunicación Breve}

Continuous decline of Toxoplasma gondii seroprevalence in hospital: a 1997-2014 longitudinal study in Paris, France. Front Microbiol 2018; 9: 2369. doi: $10.3389 /$ fmicb.2018.02369

14.- Schenone H, Sandoval L, Contreras MC, Salinas P, Rojas A. Epidemiology of toxoplasmosis in Chile. VII. Prevalence of human infection investigated by means of indirect hemagglutination reaction in the regions $\mathrm{X}, \mathrm{XI}$ and XII. Bol Chil Parasitol 1990; 45: 77-9.
15.- López J, Abarca K, Paredes P, Inzunza E. Parásitos intestinales en caninos y felinos con cuadros digestivos en Santiago, Chile: Consideraciones en Salud Pública. Rev Med Chile 2006; 134 (2): 193-200. http://dx.doi.org/10.4067/ S0034-98872006000200009.

16.- Ovalle F, García A, Thibauth J, Lorca M. Frequency of anti-Toxoplasma gondii antibodies in cats from Valdivia city, Chile. Bol Chil Parasitol 2000; 55: 94-9. 